\title{
Covering the Set of Integers by Congruence Classes of Distinct Moduli
}

\author{
By S. L. G. Choi
}

\begin{abstract}
A set of congruences is called a covering set if every integer belongs to at least one of the congruences. Erdös has raised the following question: given any number $N$, does there exist a covering set of distinct moduli such that the least of such moduli is $N$. This has been answered in the affirmative for $N$ up to 9. The aim of this paper is to show that there exists a covering set of distinct moduli the least of which is 20 . Recently, Krukenberg independently and by other methods has also obtained results up through $N=18$.
\end{abstract}

Suppose $a$ is a set of integers. The set of congruence classes $h_{i} \bmod m_{i}$ $(i=1,2, \cdots, t)$ is said to be a covering set for $a$ if every integer of $a$ belongs to at least one of the congruence classes $h_{i} \bmod m_{i}$. The question (see Problem 42 in [1]) has been raised whether for every $N$ there exists a covering set of congruence classes $h_{i} \bmod m_{i}(i=1,2, \cdots, t)$ of distinct moduli for the set of all integers such that $N \leqq m_{1}<m_{2} \cdots<m_{t}$.

For $N=3$, Davenport and Erdös, and Fried have exhibited such covering sets. Swift settled the case $N=6$, Selfridge succeeded in constructing a covering set where $N=8$, and Churchhouse (see [2]) had results for $N=9$. In this paper, we shall construct a covering set of congruence classes $h_{i} \bmod m_{i}(i=1,2, \cdots, t)$ for the set of integers where $20=m_{1}<m_{2}<\cdots<m_{t}$. It should be mentioned that, recently, Krukenberg has obtained results through $N=18$ independently and by other methods (see [3]). It is conceivable that a further elaboration of the method of the present paper is capable of producing a more favorable $N$ than 20 but the amount of computations may then become prohibitive. Finally, it should perhaps be mentioned that the question of determining if there exists a covering set of distinct odd moduli has also been raised but is still open.

Notations. We denote by $Z$ the set of integers. We denote by $\{m\}$ a congruence class modulo $m$ and, for $h \geqq 2$, we denote by $\{m\}^{h} h$ distinct congruence classes modulo $m$. Precisely which of the $m$ congruence classes modulo $m$ are implied by the notation $\{m\}$ or $\{m\}^{h}$ will be determined (in an obvious manner) by the context in which we use $\{m\}$ and $\{m\}^{n}$.

The following four theorems are essential to the underlying idea in our construction.

THEOREM 1. If the congruence class $a$ mod $m$ is covered by the congruence classes $a_{1} \bmod m_{1}, \cdots, a_{t} \bmod m_{t}$, then for any $b$, the congruence class $b \bmod m$ is covered by $c_{1} \bmod m_{1}, \cdots, c_{t} \bmod m_{t}$, where

$$
c_{i}=a_{i}+(b-a), \quad i=1,2, \cdots, t .
$$

Received December 4, 1970.

AMS 1969 subject classifications. Primary 1010, 1020.

Key words and phrases. Integers, congruences, covering set, distinct moduli.

Copyright @ 1971, American Mathematical Society 
Proof. Let $n$ be any integer belonging to $b \bmod m$. Then $n+(a-b)$ belongs to $a$ mod $m$ so that $n+(a-b)$ is contained in $a_{i} \bmod m_{i}$ for some $i$ satisfying $1 \leqq i \leqq t$. Hence, $n$ is contained in $a_{i}+(b-a) \bmod m_{i}$.

THEOREM 2. Suppose the congruence class $a$ mod $m$ can be covered by the congruence classes $a_{1} \bmod m_{1}, \cdots, a_{t} \bmod m_{t}$. Then for any natural number $c$, the congruence class $a$ $\bmod m c$ can be covered by the congruence classes $a_{1}^{\prime} \bmod m_{1} c, \cdots, a_{t}^{\prime} \bmod m_{t} c$, where

$$
a_{i}^{\prime}=c\left(a_{i}-a\right)+a, \quad i=1,2, \cdots, t .
$$

Proof. Let $n$ be any given integer belonging to $a \bmod \mathrm{cm}$. Then $n-a$ belongs to $0 \bmod \mathrm{cm}$. Since $a \bmod m$ is covered by $a_{1} \bmod m_{1}, \cdots, a_{t} \bmod m_{t}$, Theorem 1 implies that $0 \bmod m$ is covered by $\left(a_{1}-a\right) \bmod m_{1}, \cdots,\left(a_{t}-a\right) \bmod m_{t}$. Hence, 0 $\bmod \mathrm{cm}$ is covered by $c\left(a_{1}-a\right) \bmod c m_{1}, \cdots, c\left(a_{t}-a\right) \bmod c m_{t}$, since if $n^{*}$ belongs to $0 \bmod c m$, then $n^{*} / c$ belongs to $0 \bmod m$ and so there is some $i, 1 \leqq i \leqq t$, with $n^{*} / c$ contained in $\left(a_{i}-a\right) \bmod m_{i}$. Thus $n^{*}=c\left(n^{*} / c\right)$ belongs to $c\left(a_{i}-a\right) \bmod c m_{i}$. Hence, $n-a$ belongs to some congruence class $c\left(a_{i}-a\right) \bmod c m_{i}$, where $i$ satisfies $1 \leqq i \leqq t$ so that $n$ belongs to $c\left(a_{i}-a\right)+a \bmod c m_{i}$.

THEOREM 3. Let $n$ be a natural number. Then the congruence class $0 \bmod n$ is covered by the congruence classes $\{2 n\},\{3 n\},\left\{2^{2} n\right\},\{2 \cdot 3 n\},\left\{2^{2} \cdot 3 n\right\}$.

Proof. We note that $0 \bmod 1$ is the union of $1 \bmod 2,0 \bmod 4$ and $2 \bmod 4$. Now $0 \bmod 4$ is the union of $0 \bmod 2^{2} \cdot 3,4 \bmod 2^{2} \cdot 3$ and $8 \bmod 2^{2} \cdot 3$. Since $0 \bmod 2^{2} \cdot 3$ is contained in $0 \bmod 3$ and $4 \bmod 2^{2} \cdot 3$ in $4 \bmod 2 \cdot 3$, we see that $0 \bmod 4$ is covered by 0 $\bmod 3,4 \bmod 2 \cdot 3$ and $8 \bmod 2^{2} \cdot 3$. Hence, the congruence classes $1 \bmod 2,2 \bmod 4$, $0 \bmod 3,4 \bmod 6$ and $8 \bmod 12$ constitute a covering set for $0 \bmod 1$. Finally, Theorem 3 is a direct consequence of this covering set and Theorem 2 .

COROLlaRY. For any $r \geqq 1$, the set of congruence classes $\{2 n\},\left\{2^{2} n\right\}, \cdots,\left\{2^{r} n\right\}$, $\left\{3 \cdot 2^{r-1} n\right\},\left\{2^{r+1} n\right\},\left\{2^{r} \cdot 3 n\right\},\left\{2^{r+1} \cdot 3 n\right\}$ covers the congruence class $0 \bmod n$.

Proof. For $r=1$, the Corollary is simply a restatement of Theorem 3; and for $r \geqq 2$, the congruence classes $\{2\},\left\{2^{2}\right\}, \cdots,\left\{2^{r-2}\right\},\left\{2^{r-1}\right\}^{2}$ form a covering set for the integers. Theorems 3 and 1 imply a class $\left\{2^{r-1}\right\}$ can be covered by $\left\{2^{r-1} \cdot 2\right\}$, $\left\{2^{r-1} \cdot 3\right\},\left\{2^{r-1} \cdot 2^{2}\right\},\left\{2^{r-1} \cdot 2 \cdot 3\right\},\left\{2^{r-1} \cdot 2^{2} \cdot 3\right\}$; and now we obtain the Corollary by applying Theorem 2 .

THEOREM 4. Suppose the set $y$ of integers can be covered by $\left\{m_{1}\right\}^{\alpha_{1}},\left\{m_{2}\right\}^{\alpha_{2}}, \cdots$, $\left\{m_{t}\right\}^{\alpha_{t}}$ where $m_{1}<m_{2}<\cdots<m_{t}$ and $\alpha_{i}=1$ or $2(i=1,2, \cdots, t)$. Suppose further that for every pair $i, j$ satisfying $1 \leqq j<i \leqq t$, the relation $m_{i} m_{i}^{-1}=2^{8}$ cannot hold for any natural number $s$ unless $\alpha_{i}=1$. Then there exists a covering set of congruence classes $\left\{n_{1}\right\},\left\{n_{2}\right\}, \cdots,\left\{n_{l}\right\}$ of distinct moduli for $Y$ where

$$
m_{1}=n_{1}<n_{2}<\cdots<n_{l} .
$$

Proof. We may suppose that $\alpha_{i}=2$ for at least one $i$ satisfying $1 \leqq i \leqq t$ since otherwise we already have the theorem. We denote by $D$ the set of integers $m_{1}, m_{2}, \cdots$, $m_{t}$; and by $\mathcal{C}$ the subset of $\mathfrak{D}$ consisting of those integers such that the corresponding $\alpha_{i}$ appearing in $\left\{m_{i}\right\}^{\alpha_{i}}$ is equal to 2 . We then partition $\mathcal{C}$ into nonempty disjoint subsets $\mathfrak{C}_{1}, \cdots, \mathfrak{C}_{v}$, where $\mathfrak{C}_{i}(i=1,2, \cdots, v)$ consists of those integers of $\mathfrak{e}$ such that the exact power of 3 dividing them is $k_{i}$, where $0 \leqq k_{1}<k_{2}<\cdots<k_{v}$. For $i=1,2, \cdots, v$, we denote any integer in $\mathcal{C}_{i}$ by $c_{i}$ and we denote by $c_{i}^{\min }$ and $c_{i}^{\max }$ the least and the largest of the integers in $\mathfrak{C}_{i}$. Clearly, we have 


$$
\bigcup_{i=1}^{i}\left\{m_{i}\right\}^{\alpha_{i}}=\left(\bigcup_{i=1} \bigcup_{c_{i} \in \mathfrak{e}_{i}}\left\{c_{i}\right\}\right) \cup\left(\bigcup_{i=1}^{i}\left\{m_{i}\right\}\right)
$$

Now, as is possible by Theorem 1 and the Corollary of Theorem 3, we can cover $\left\{c_{i}\right\}$ by

$$
\left\{2 c_{i}\right\}, \cdots,\left\{2^{r_{i}} c_{i}\right\}, \quad\left\{3 \cdot 2^{r_{i}-1} c_{i}\right\}, \quad\left\{2^{r_{i}+1} c_{i}\right\}, \quad\left\{2^{r_{i}} \cdot 3 \cdot c_{i}\right\}, \quad\left\{2^{r_{i}+1} \cdot 3 \cdot c_{i}\right\} ;
$$

and we choose $r_{i}$ inductively so that

$$
2^{r \cdot} c_{v}^{\text {in }}>m_{t}
$$

and

$$
2^{r i} c_{i}^{\min }>2^{r_{i+1}+1} \cdot 3 \cdot c_{i+1}^{\max } \quad(i=v-1, v-2, \cdots, 1) .
$$

On defining $D_{i}$ and $D_{i}^{*}$ by

$$
D_{i}=\bigcup_{c_{i} \in \mathfrak{e}_{i}}\left\{2 c_{i}, \cdots, 2^{r_{i}} c_{i}, 3 \cdot 2^{r_{i}-1} c_{i}, 2^{r_{i+1}} c_{i}, 2^{r_{i}} \cdot 3 \cdot c_{i}, 2^{r_{i+1}} \cdot 3 \cdot c_{i}\right\}
$$

and

$$
\mathscr{D}_{i}^{*}=\bigcup_{d_{i} \in \mathbb{D}_{i}}\left\{d_{i}\right\},
$$

we obviously have

$$
\bigcup_{c_{i} \in \mathfrak{e}_{i}}\left\{c_{i}\right\} \subset \bigcup_{d_{i} \in \mathcal{D}_{i}}\left\{d_{i}\right\}=\mathscr{D}_{i}^{*},
$$

so that, in view of (1), we have

$$
\bigcup_{i=1}^{t}\left\{m_{i}\right\}^{\alpha_{i}} \subset \mathcal{D}_{1}^{*} \cup \ldots \cup \mathcal{D}_{v}^{*} \cup\left\{m_{1}\right\} \cup \cdots \cup\left\{m_{t}\right\} .
$$

To establish the theorem, it thus suffices to show the following:

(i) For any given $i$ satisfying $1 \leqq i \leqq v$, the integers in $D_{i}$ are all distinct.

(ii) If $x \in D_{i}$ and $y \in D_{i}$ where $i \neq j$, then $x \neq y$.

(iii) If $x \in D_{i}$ where $i$ satisfies $1 \leqq i \leqq v$, then $x \neq m_{i}$ for any $j$ satisfying $1 \leqq j \leqq t$.

We first show that (i) holds. Suppose $2^{\alpha_{1}} 3^{\beta_{1}} s_{1}, 2^{\alpha_{2}} 3^{\beta_{2}} s_{2}$ are two integers belonging to $D_{i}$, where $\alpha_{1} \geqq 0, \alpha_{2} \geqq 0, \beta_{1} \geqq 0, \beta_{2} \geqq 0$ and $s_{1}, s_{2} \in \mathcal{C}_{i}$. The same exact power of 3 divides both $s_{1}$ and $s_{2}$. We may further assume that $s_{1} \neq s_{2}$, so that without loss of generality $s_{1}>s_{2}$ (since if $s_{1}=s_{2}$, then, clearly, $2^{\alpha_{2}} 3^{\beta_{1}} s_{1}, 2^{\alpha_{2}} 3^{\beta_{3}} s_{2}$ are different). Then $s_{1}=2^{s} s_{2}$ cannot hold for a natural number $s$ since there are two congruence classes of modulus $s_{2}$ in the set $\left\{m_{1}\right\}^{\alpha_{1}}, \cdots,\left\{m_{t}\right\}^{\alpha_{t}}$. Therefore, there exists a prime $p(\neq 2,3)$ so that the exact power of $p$ dividing $s_{1}$ is not equal to that dividing $s_{2}$. Thus, $2^{\alpha_{1}} 3^{\beta_{2}} s_{1} \neq 2^{\alpha} 3^{\beta_{3}} s_{2}$, as required.

Next, we show that (ii) holds. We may suppose without loss of generality $i>j$. Then (3) implies that $y$ is either greater than any integer in $D_{i}$ and in this case we already have the validity of (ii), or else $y=2^{k} c_{j}$ where $k>0$ and $c_{i} \in \mathfrak{e}_{i}$. Since $x$ is of the type $2^{\alpha} 3^{\beta} c_{i}$ where $\alpha \geqq 0, \beta \geqq 0$ and $c_{i} \in \mathcal{C}_{i}$, clearly, $y$ cannot be equal to $x$, the exact power of 3 dividing $x$ being clearly greater than the corresponding one for $y$. 
Finally, we show that (iii) is true. In view of (2) and (3), $x$ is either greater than $m_{t}$ (and in this case obviously $x$ and $m_{i}$ cannot be equal) or $x$ is of the type $2^{k} c_{i}$ where $k>0$ and $c_{i} \in \mathcal{C}_{i}$. Now, the equality between $x$ and $m_{i}$ would imply that $m_{i}=2^{k} m_{l}$ since $c_{i}$ is equal to some $m_{l}$ where $l$ satisfies $1 \leqq l \leqq t$. This is clearly an impossibility since $\alpha_{l}=2$.

Prior to the actual construction of the required covering set for $Z$, we shall here introduce some further definitions and notations.

Definition 1. Let $a$ be a set of integers. Then the set of congruence classes $\left\{m_{1}\right\}^{\alpha_{1}}, \cdots,\left\{m_{t}\right\}^{\alpha_{t}}$, where $m_{1}<\cdots<m_{t}$ and $\alpha_{i}=1$ or $2(i=1,2, \cdots, t)$, is said to be $a$-good if $a$ is covered by $\left\{m_{1}\right\}^{\alpha_{1}}, \cdots,\left\{m_{t}\right\}^{\alpha_{t}}$ and if for every pair $i, j$, satisfying $1 \leqq j<i \leqq t$, the relation $m_{i} m_{j}^{-1}=2^{s}$ cannot hold for a natural number $s$ unless $\alpha_{i}=1$.

In view of Theorem 4, the existence of an a-good set implies the existence of a covering set of congruence classes of distinct moduli for $a$ such that the least modulus of the latter is not smaller than the least modulus of the former.

Definition 2. An integer $n$ is said to belong to $q_{1} \cdots q_{r}$, where $q_{i}$ is the $i$ th largest prime divisor of $n(i=1,2, \cdots, r)$. Thus, if $q_{1}>q_{2}>\cdots>q_{s}$ are all the distinct prime divisors of $n$, then $n$ belongs to each of the $s$ integers

$$
q_{1} q_{2} \cdots q_{r} \quad(r=1,2, \cdots, s) .
$$

Definition 3. Suppose $S$ is a set of congruence classes. Then we define the index of $S$ to be the smallest of the moduli of the congruence classes in $\delta$.

In view of the above definitions and Theorem 4, our aim in this paper is to show that there is a $Z$-good set of index 20 .

It is almost self-evident that if $X$ is $a$-good and $Y$ is $B$-good, and if a certain prime divides each of the moduli in $x$ but none of those in $y$, then $x \cup y$ is $a \cup B$-good. On making use of this fact, we see that the following theorems (Theorems 5-13) clearly imply that $\bigcup_{i=1}^{9} Q_{i}$ is $Z$-good. That $\bigcup_{i=1}^{9} Q_{i}$ is of index 20 is easily verified. Perhaps, we should mention that a little scrutiny is required to verify that $Q_{3}$, together with the congruence classes in (I) of Theorem 8, form a $\left\{2^{4}\right\} \cup \mathcal{S}$-good set, where $S$ is the set covered by (I). Finally, we mention that the parameter $k$ appearing in the following theorems stands for any given integer $>111$.

THEOREM 5. A Z-good set is the set of congruence classes $\left\{2^{2}\right\},\left\{2^{3}\right\},\left\{2^{4}\right\},\left\{2^{5}\right\}^{2}$, $\{2 \cdot 3\},\left\{2^{2} \cdot 3\right\},\left\{2^{3} \cdot 3\right\}^{2},\left\{2 \cdot 3^{2}\right\},\left\{2^{2} \cdot 3^{2}\right\}^{2},\left\{3^{3}\right\},\left\{2 \cdot 3^{3}\right\}^{2}$. We denote by $a_{1}$ the set $\left\{2^{5}\right\}^{2},\left\{2^{3} \cdot 3\right\}^{2},\left\{2^{2} \cdot 3^{2}\right\}^{2},\left\{3^{3}\right\},\left\{2 \cdot 3^{3}\right\}^{2}$.

THEOREM 6.* There exists $a\left\{2^{2}\right\}$-good set $Q_{2}$ of congruence classes whose moduli all belong to 5, namely

$$
\begin{gathered}
\left\{3^{2} \cdot 5\right\},\left\{2 \cdot 3^{\beta} \cdot 5\right\}_{\beta=1,2,3}, \\
\left\{2^{\alpha} \cdot 3^{\beta} \cdot 5^{\gamma}\right\}_{\alpha=0,1 ; \beta=0,1,2,3 ; \gamma-2,3,4}, \quad\left\{2^{2} \cdot 3^{\beta} \cdot 5^{\gamma}\right\}_{\beta-0,1,2,3 ; \gamma-1,2,3,4}^{2} .
\end{gathered}
$$

THEOREM 7. There exists $a\left\{2^{4}\right\}$-good set $a_{3}$ of congruence classes whose moduli all belong to 7, namely

* It will be noticed that the moduli listed in Theorems 6-13 invariably include some which are not actually used in the proofs, and this is done for the convenience of listing. It is also clear that these moduli in excess in no way affect the final outcome in our present approach concerning the computation of the smallest modulus used in a covering set of distinct moduli. 


$$
\left\{2 \cdot 7^{2}\right\}, \quad\left\{2^{\alpha} \cdot 3^{\beta} \cdot 5^{\gamma} \cdot 7^{\delta}\right\}_{\alpha-2,3 ; \beta=0,1 ; \gamma=0,1 ; 8=1,2}
$$

where we exclude the case $\alpha=2, \beta=1, \gamma=0$, and

$$
\left\{2^{4} \cdot 3^{\beta} \cdot 5^{\gamma} \cdot 7^{\delta}\right\}_{\beta=0,1 ; \gamma=0,1 ; \delta-1,2}^{2} \text {. }
$$

THEOREM 8. There exists $a\{2 \cdot 3\}$-good set $a_{4}$ of congruence classes whose moduli all belong to $7,11 \cdot 7,37 \cdot 11 \cdot 7,13 \cdot 7,13 \cdot 11 \cdot 7,37 \cdot 13 \cdot 11 \cdot 7$ or $37 \cdot 13 \cdot 7$. These congruence classes fall into 3 main categories, namely,

$$
\begin{aligned}
& \left\{7^{2}\right\}, \quad\left\{2 \cdot 3^{\beta} \cdot 7^{3}\right\}_{\beta=0,1,2}^{2}, \quad\left\{3^{\beta} 7^{3}\right\}_{\beta=0,1,2}, \\
& \left\{2^{\alpha} \cdot 3 \cdot 7^{\gamma}\right\}_{\alpha=0,1,2 ; \gamma=1,2}, \\
& \left\{2^{\alpha} \cdot 3^{\beta} \cdot 5 \cdot 7^{\gamma}\right\}_{\alpha=0,1 ; \beta=0,1 ; \gamma=1,2}, \\
& \left\{3^{2} \cdot 5^{\delta} \cdot 7^{\gamma}\right\}, \quad\left\{2 \cdot 3^{2} \cdot 5^{\delta} \cdot 7^{\gamma}\right\}_{\delta=0,1 ; \gamma=1,2}^{2} .
\end{aligned}
$$$$
\left\{3^{\alpha} \cdot 5^{\beta} \cdot 7^{\gamma} \cdot 11^{\delta}\right\}, \quad\left\{2 \cdot 3^{\alpha} \cdot 5^{\beta} \cdot 7^{\gamma} \cdot 11^{\delta}\right\}_{\alpha}^{2}
$$

$$
\begin{aligned}
&\left\{2 \cdot 3 \cdot 5^{\beta} \cdot 7 \cdot 11^{\gamma} \cdot 37\right\}_{\beta=1,2}, \cdots, 74 ; \gamma=1,2, \cdots, k \\
&\left\{2 \cdot 3 \cdot 7 \cdot 11^{\delta} \cdot 37\right\}_{\delta=1,2, \cdots, 37} . \\
&\left\{2^{\alpha} \cdot 3^{\beta} \cdot 5^{\delta} \cdot 7^{\gamma} \cdot 11^{\epsilon} \cdot 13\right\}_{\alpha=0,1 ; \beta=0,1, \cdots, k ; \delta=0,1 ; \gamma=1,2 ; \epsilon=0,1}, \\
&\left\{2^{2} \cdot 3^{\beta} \cdot 5^{\delta} \cdot 11^{\epsilon} \cdot 7^{\gamma} \cdot 13\right\}_{\beta=0,1, \cdots, k ; \delta=0,1 ; \gamma=1,2 ; \epsilon=0,1}^{2}, \\
&\left\{2^{2} \cdot 3^{\beta} \cdot 5^{\delta} \cdot 7^{\gamma} \cdot 13 \cdot 11^{\epsilon} \cdot 37\right\}_{\beta=1,2, \cdots, 74 ; \delta=0,1 ; \epsilon=0,1 ; \gamma=1,2} .
\end{aligned}
$$

THEOREM 9. There exists $a\left\{2^{2} \cdot 3\right\}$-good set $a_{5}$ of congruence classes whose moduli all belong to 11 or $11 \cdot 37$ but none of these moduli is divisible by 7 . These congruence classes are

$$
\left\{2^{\alpha} \cdot 3^{\beta} \cdot 5^{\gamma} \cdot 11\right\}, \quad\left\{2^{2} \cdot 3^{\beta} \cdot 5^{\gamma} \cdot 11\right\}^{2},
$$

where $\alpha=0,1 ; \beta=0,1, \cdots, k ; \gamma=0,1$ but the case $\alpha=\beta=\gamma=0$ is excluded, and

$$
\left\{2^{2} \cdot 3^{\beta} \cdot 5^{\gamma} \cdot 37 \cdot 11\right\}_{\beta=1,2, \cdots, 74 ; \gamma=0,1} .
$$

THEOREM 10. There exists $a\left\{2 \cdot 3^{2}\right\}$-good set $a_{6}$ of congruence classes whose moduli all belong to 13 or 13.37 but none of these moduli is divisible by 7 . These congruence classes are

$$
\left\{3^{\alpha} \cdot 5^{\beta} \cdot 11^{\gamma} \cdot 13^{\delta}\right\}, \quad\left\{2 \cdot 3^{\alpha} \cdot 5^{\beta} \cdot 11^{\gamma} \cdot 13^{\delta}\right\}^{2},
$$

where $\alpha=0,1,2,3 ; \beta=0,1 ; \gamma=0,1 ; \delta=1,2$, but the case $\alpha=\beta=\gamma=0$, $\delta=1$ is excluded.

THEOREM 11. There exists $a\left\{2^{3} \cdot 3\right\}$-good set $a_{7}$ of congruence classes whose moduli all belong to 17 or $17 \cdot 37$. They are

$$
\left\{2^{\alpha} \cdot 3^{\beta} \cdot 5^{\gamma} \cdot 7^{\delta} \cdot 11^{\epsilon} \cdot 13^{\phi} \cdot 17\right\}, \quad\left\{2^{3} \cdot 3^{\beta} \cdot 5^{\gamma} \cdot 7^{\delta} \cdot 11^{\epsilon} \cdot 13^{\phi} \cdot 17\right\}^{2},
$$

where $\alpha=0,1,2 ; \beta=0,1,2,3 ; \gamma=0,1,2, \cdots, k ; \delta=0,1 ; \epsilon=0,1 ; \phi=0,1$ but we exclude the case $\alpha=\beta=\gamma=\delta=\epsilon=\phi=0$, and

$$
\left\{2^{3} \cdot 3 \cdot 5^{\gamma} \cdot 37 \cdot 17\right\}_{\gamma-1,2, \cdots, 111} \text {. }
$$

THEOREM 12. There exists $a\left\{2^{3} \cdot 3\right\}$-good set $a_{8}$ of congruence classes whose moduli all belong to 19 or $37 \cdot 19$. They are 


$$
\left\{2^{\alpha} \cdot 3^{\beta} \cdot 5^{\gamma} \cdot 7^{\delta} \cdot 11^{\epsilon} \cdot 13^{\phi} \cdot 17^{\psi} \cdot 19\right\}, \quad\left\{2^{3} \cdot 3^{\beta} \cdot 5^{\gamma} \cdot 7^{\delta} \cdot 11^{\epsilon} \cdot 13^{\phi} \cdot 17^{\psi} \cdot 19\right\}^{2},
$$

where $\alpha=0,1,2 ; \beta=0,1,2,3 ; \gamma=0,1, \cdots, k ; \delta=0,1 ; \epsilon=0,1 ; \phi=0,1$; $\psi=0,1$ but we exclude the case $\alpha=\beta=\gamma=\delta=\epsilon=\phi=\psi=0$, and

$$
\left\{2^{3} \cdot 3 \cdot 5^{\gamma} \cdot 17^{\psi} \cdot 37 \cdot 19\right\}_{\gamma-1,2, \cdots, 111 ; \psi=0,1} .
$$

THEOREM 13. There exists $a\left\{2^{3} \cdot 3\right\}$-good set $a_{9}$ of congruence classes whose moduli all belong to $23,23 \cdot 37,29$ or 31 . They are

$$
\left\{2^{\alpha} \cdot 3^{\beta} \cdot 5^{\gamma} \cdot 7^{\delta} \cdot 11^{\epsilon} \cdot 13^{\phi} \cdot 17^{\psi} \cdot 19^{\lambda} \cdot 23\right\}, \quad\left\{2^{3} \cdot 3^{\beta} \cdot 5^{\gamma} \cdot 7^{\delta} \cdot 11^{\epsilon} \cdot 13^{\phi} \cdot 17^{\psi} \cdot 19^{\lambda} \cdot 23\right\}^{2}
$$

where $\alpha=0,1,2 ; \beta=0,1,2,3 ; \gamma=0,1, \cdots, k ; \delta=0,1 ; \epsilon=0,1 ; \phi=0,1$; $\psi=0,1 ; \lambda=0,1$,

$$
\left\{2^{3} \cdot 3 \cdot 5^{\gamma} \cdot 17^{\psi} \cdot 19^{\lambda} \cdot 23 \cdot 37\right\}_{\gamma-1,2, \cdots, 111 ; \psi-0,1 ; \lambda=0,1}
$$

and

$$
\left\{2^{\alpha} \cdot 3^{\beta} \cdot 5^{\gamma} \cdot 7^{\delta} \cdot 11^{\epsilon} \cdot 23^{\mu} \cdot a\right\}, \quad\left\{2^{3} \cdot 3^{\beta} \cdot 5^{\gamma} \cdot 7^{\delta} \cdot 23^{\mu} \cdot a\right\}^{2}
$$

where $\alpha=0,1,2 ; \beta=0,1,2,3 ; \gamma=0,1 ; \delta=0,1 ; a=29,31 ; \mu=0,1 ; \epsilon=0,1$.

We now proceed to the proofs of Theorems $5-13$. The steps in the proofs will be presented in tabulated form. In each of these tables, the right-hand column will consist of covering sets of congruence classes for the corresponding congruence classes in the left column.

Proof of Theorem 5.

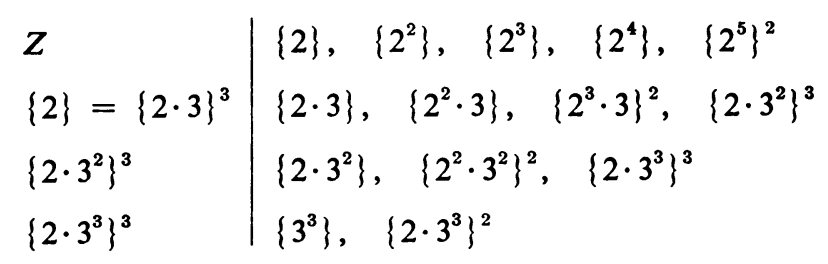

Proof of Theorem 6.

$$
\begin{array}{l|l}
\left\{2^{2}\right\}=\left\{2^{2} \cdot 5\right\}^{5} & \left\{2^{2} \cdot 5\right\}^{2}\left\{2^{2} \cdot 3 \cdot 5\right\}^{6}\left\{2^{2} \cdot 5^{2}\right\}^{5} \\
\left\{2^{2} \cdot 3 \cdot 5\right\}^{6} & \{2 \cdot 3 \cdot 5\}\left\{2^{2} \cdot 3 \cdot 5\right\}^{2}\left\{2^{2} \cdot 3^{2} \cdot 5\right\}^{6}\left\{2^{2} \cdot 3 \cdot 5^{2}\right\}^{5} \\
\left\{2^{2} \cdot 3^{2} \cdot 5\right\}^{6} & \left\{3^{2} \cdot 5\right\}\left\{2 \cdot 3^{2} \cdot 5\right\}\left\{2^{2} \cdot 3^{2} \cdot 5\right\}^{2}\left\{2^{2} \cdot 3^{3} \cdot 5\right\}^{3}\left\{2^{2} \cdot 3^{2} \cdot 5^{2}\right\}^{5} \\
\left\{2^{2} \cdot 3^{3} \cdot 5\right\}^{3} & \left\{2 \cdot 3^{3} \cdot 5\right\}\left\{2^{2} \cdot 3^{3} \cdot 5\right\}^{2} \\
\left\{2^{2} \cdot 5^{2}\right\}^{5} & \left\{2^{\alpha} \cdot 5^{2}\right\}_{\alpha=0,1}\left\{2^{2} \cdot 5^{2}\right\}^{2}\left\{2^{2} \cdot 5^{3}\right\}^{5} \\
\left\{2^{2} \cdot 3 \cdot 5^{2}\right\}^{5} & \left\{2^{\alpha} \cdot 3 \cdot 5^{2}\right\}_{\alpha=0,1}\left\{2^{2} \cdot 3 \cdot 5^{2}\right\}^{2}\left\{2^{2} \cdot 3 \cdot 5^{3}\right\}^{5} \\
\left\{2^{2} \cdot 3^{2} \cdot 5^{2}\right\}^{5} & \left\{2^{\alpha} \cdot 3^{2} \cdot 5^{2}\right\}_{\alpha=0,1}\left\{2^{2} \cdot 3^{2} \cdot 5^{2}\right\}^{2}\left\{2^{2} \cdot 3^{3} \cdot 5^{2}\right\}^{3} \\
\left\{2^{2} \cdot 3^{3} \cdot 5^{2}\right\}^{3} & \left\{2 \cdot 3^{3} \cdot 5^{2}\right\}\left\{2^{2} \cdot 3^{3} \cdot 5^{2}\right\}^{2} \\
\left\{2^{2} \cdot 5^{3}\right\}^{5} & \left\{2^{\alpha} \cdot 5^{3}\right\}_{\alpha=0,1}\left\{2^{2} \cdot 5^{3}\right\}^{2}\left\{2^{2} \cdot 5^{4}\right\}^{5} \\
\left\{2^{2} \cdot 3 \cdot 5^{3}\right\}^{5} & \left\{2^{\alpha} \cdot 3 \cdot 5^{3}\right\}_{\alpha=0,1}\left\{2^{2} \cdot 3 \cdot 5^{3}\right\}^{2}\left\{2^{2} \cdot 3^{2} \cdot 5^{3}\right\}^{3} \\
\left\{2^{2} \cdot 3^{2} \cdot 5^{3}\right\}^{3} & \left\{2 \cdot 3^{2} \cdot 5^{3}\right\}\left\{2^{2} \cdot 3^{2} \cdot 5^{3}\right\}^{2}
\end{array}
$$




$$
\begin{array}{l|l}
\left\{2^{2} \cdot 5^{4}\right\}^{5} & \left\{2^{\alpha} \cdot 5^{4}\right\}_{\alpha=0,1}\left\{2^{2} \cdot 5^{4}\right\}^{2}\left\{2^{2} \cdot 3 \cdot 5^{4}\right\}^{3} \\
\left\{2^{2} \cdot 3 \cdot 5^{4}\right\}^{3} & \left\{2 \cdot 3 \cdot 5^{4}\right\}\left\{2^{2} \cdot 3 \cdot 5^{4}\right\}^{2}
\end{array}
$$

Proof of Theorem 7.

$$
\begin{array}{l|l}
\left\{2^{4}\right\}=\left\{2^{4} \cdot 7\right\}^{7} & \left\{2^{\alpha} \cdot 7\right\}_{\alpha=2,3}\left\{2^{4} \cdot 7\right\}^{2}\left\{2^{4} \cdot 3 \cdot 7\right\}^{3}\left\{2^{4} \cdot 5 \cdot 7\right\}^{5}\left\{2^{4} \cdot 7^{2}\right\}^{7} \\
\left\{2^{4} \cdot 3 \cdot 7\right\}^{3} & \left\{2^{3} \cdot 3 \cdot 7\right\}\left\{2^{4} \cdot 3 \cdot 7\right\}^{2} \\
\left\{2^{4} \cdot 5 \cdot 7\right\}^{5} & \left\{2^{\alpha} \cdot 5 \cdot 7\right\}_{\alpha=2,3}\left\{2^{4} \cdot 5 \cdot 7\right\}^{2}\left\{2^{4} \cdot 3 \cdot 5 \cdot 7\right\}^{3} \\
\left\{2^{4} \cdot 3 \cdot 5 \cdot 7\right\}^{3} & \left\{2^{\alpha} \cdot 3 \cdot 5 \cdot 7\right\}_{\alpha=2,3,4} \\
\left\{2^{4} \cdot 7^{2}\right\}^{7} & \left\{2^{\alpha} \cdot 7^{2}\right\}_{\alpha=1,2,3}\left\{2^{4} \cdot 7^{2}\right\}^{2}\left\{2^{4} \cdot 3 \cdot 7\right\}^{3}\left\{2^{4} \cdot 5 \cdot 7^{2}\right\}^{5} \\
\left\{2^{4} \cdot 3 \cdot 7^{2}\right\}^{3} & \left\{2^{3} \cdot 3 \cdot 7^{2}\right\}\left\{2^{4} \cdot 3 \cdot 7^{2}\right\}^{2} \\
\left\{2^{4} \cdot 5 \cdot 7^{2}\right\}^{5} & \left\{2^{\alpha} \cdot 5 \cdot 7^{2}\right\}_{\alpha=2,3}\left\{2^{4} \cdot 5 \cdot 7^{2}\right\}^{2}\left\{2^{4} \cdot 3 \cdot 5 \cdot 7^{2}\right\}^{3} \\
\left\{2^{4} \cdot 3 \cdot 5 \cdot 7^{2}\right\}^{3} & \left\{2^{\alpha} \cdot 3 \cdot 5 \cdot 7^{2}\right\}_{\alpha=2,3,4}
\end{array}
$$

Proof of Theorem 8.

Step 1.

$$
\begin{array}{l|l}
\{2 \cdot 3\}=\{2 \cdot 3 \cdot 7\}^{7} & \begin{array}{l}
\left\{2^{\alpha} \cdot 3 \cdot 7\right\}_{\alpha=0,1,2}\left\{2^{2} \cdot 3 \cdot 7 \cdot 13\right\}^{13}\left\{2 \cdot 3^{2} \cdot 7\right\}^{3} \\
\{2 \cdot 3 \cdot 5 \cdot 7\}^{5}\left\{2 \cdot 3 \cdot 7^{2}\right\}^{7}\{2 \cdot 3 \cdot 7 \cdot 11\}^{11}
\end{array} \\
\left\{2 \cdot 3^{2} \cdot 7\right\}^{3} & \left\{3^{2} \cdot 7\right\}\left\{2 \cdot 3^{2} \cdot 7\right\}^{2} \\
\{2 \cdot 3 \cdot 5 \cdot 7\}^{5} & \left\{2^{\alpha} \cdot 3^{\beta} \cdot 5 \cdot 7\right\}_{\alpha=0,1 ; \beta=0,1}\left\{2 \cdot 3^{2} \cdot 5 \cdot 7\right\}^{3} \\
\left\{2 \cdot 3^{2} \cdot 5 \cdot 7\right\}^{3} & \left\{3^{2} \cdot 5 \cdot 7\right\}\left\{2 \cdot 3^{2} \cdot 5 \cdot 7\right\}^{2} \\
\left\{2 \cdot 3 \cdot 7^{2}\right\}^{7} & \left\{3^{\alpha} \cdot 7^{2}\right\}_{\alpha=0,1}\left\{2 \cdot 3 \cdot 7^{2}\right\}\left\{2^{2} \cdot 3 \cdot 7^{2}\right\}\left\{2^{2} \cdot 3 \cdot 7^{2} \cdot 13\right\}^{13} \\
& \left\{2 \cdot 3^{2} \cdot 7^{2}\right\}^{3}\left\{2 \cdot 3 \cdot 5 \cdot 7^{2}\right\}^{5}\left\{2 \cdot 3 \cdot 7^{3}\right\}^{7} \\
\left\{2 \cdot 3^{2} \cdot 7^{2}\right\}^{3} & \left\{3^{2} \cdot 7^{2}\right\}\left\{2 \cdot 3^{2} \cdot 7^{2}\right\}^{2} \\
\left\{2 \cdot 3 \cdot 5 \cdot 7^{2}\right\}^{5} & \left\{2^{\alpha} \cdot 3^{\beta} \cdot 5 \cdot 7^{2}\right\}_{\alpha=0,1 ; \beta=0,1}\left\{2 \cdot 3^{2} \cdot 5 \cdot 7^{2}\right\}^{3} \\
\left\{2 \cdot 3^{2} \cdot 5 \cdot 7^{2}\right\}^{3} & \left\{3^{2} \cdot 5 \cdot 7^{2}\right\}\left\{2 \cdot 3^{2} \cdot 5 \cdot 7^{2}\right\}^{2} \\
\left\{2 \cdot 3 \cdot 7^{3}\right\}^{7} & \left\{3^{\beta} \cdot 7^{3}\right\}_{\beta-0,1}\left\{2 \cdot 3^{\beta} \cdot 7^{3}\right\}_{\beta=0,1}^{2}\left\{2 \cdot 3^{2} \cdot 7^{3}\right\}^{3} \\
\left\{2 \cdot 3^{2} \cdot 7^{3}\right\}^{3} & \left\{3^{2} \cdot 7^{3}\right\}\left\{2 \cdot 3^{2} \cdot 7^{3}\right\}^{2}
\end{array}
$$

A comparison of the moduli listed in Step 1 and in (I) of Theorem 8 reveals that a covering set of $\{2 \cdot 3\}$ consists of all the classes in (I) together with $\left\{2^{2} \cdot 3 \cdot 7 \cdot 13\right\}^{13}$, $\left\{2^{2} \cdot 3 \cdot 7^{2} \cdot 13\right\}^{13}$ and $\{2 \cdot 3 \cdot 7 \cdot 11\}^{11}$. It is the aim of Steps 2 and 3 to replace these latter congruence classes by appropriate congruence classes which cover them.

Step 2.

$$
\begin{array}{l|l}
\{2 \cdot 3 \cdot 7 \cdot 11\}^{11} & \begin{array}{l}
\left\{3^{\beta} \cdot 7 \cdot 11\right\}_{\beta=0,1}\left\{2 \cdot 3^{\beta} \cdot 7 \cdot 11\right\}_{\beta=0.1}^{2}\left\{2 \cdot 3^{2} \cdot 7 \cdot 11\right\}^{3} \\
\{2 \cdot 3 \cdot 5 \cdot 7 \cdot 11\}^{10}\left\{2 \cdot 3 \cdot 7^{2} \cdot 11\right\}^{7}\left\{2 \cdot 3 \cdot 7 \cdot 11^{2}\right\}^{11}
\end{array} \\
\left\{2 \cdot 3^{2} \cdot 7 \cdot 11\right\}^{3} & \left\{3^{2} \cdot 7 \cdot 11\right\}\left\{2 \cdot 3^{2} \cdot 7 \cdot 11\right\}^{2}
\end{array}
$$




$$
\begin{aligned}
& \left\{2 \cdot 3 \cdot 7^{2} \cdot 11\right\}^{7} \\
& \{2 \cdot 3 \cdot 5 \cdot 7 \cdot 11\}^{10} \\
& \left\{2 \cdot 3^{2} \cdot 5 \cdot 7 \cdot 11\right\}^{3} \\
& \left\{2 \cdot 3 \cdot 5 \cdot 7^{2} \cdot 11\right\}^{7} \\
& \left\{2 \cdot 3 \cdot 5^{2} \cdot 7 \cdot 11\right\}^{10} \\
& \text {............ } \\
& \left\{2 \cdot 3 \cdot 5^{k} \cdot 7 \cdot 11\right\}^{10} \\
& \left\{2 \cdot 3 \cdot 5^{k} \cdot 7 \cdot 11 \cdot 37\right\}^{74} \\
& \left\{2 \cdot 3 \cdot 7 \cdot 11^{2}\right\}^{11} \\
& \left\{2 \cdot 3 \cdot 7 \cdot 11^{k}\right\}^{11} \\
& \left\{2 \cdot 3 \cdot 7 \cdot 11^{k} \cdot 37\right\}^{37} \\
& \left\{3^{\beta} \cdot 7^{2} \cdot 11\right\}_{\beta=0,1,2}\left\{2 \cdot 3^{\beta} \cdot 7^{2} \cdot 11\right\}_{\beta=0,1,2}^{2} \\
& \left\{3^{\beta} \cdot 5 \cdot 7 \cdot 11\right\}_{\beta=0.1}\left\{2 \cdot 3^{\beta} \cdot 5 \cdot 7 \cdot 11\right\}_{\beta=0.1}^{2}\left\{2 \cdot 3^{2} \cdot 5 \cdot 7 \cdot 11\right\}^{3} \\
& \left\{2 \cdot 3 \cdot 5 \cdot 7^{2} \cdot 11\right\}^{7}\left\{2 \cdot 3 \cdot 5^{2} \cdot 7 \cdot 11\right\}^{10} \\
& \left\{3^{2} \cdot 5 \cdot 7 \cdot 11\right\}\left\{2 \cdot 3^{2} \cdot 5 \cdot 7 \cdot 11\right\}^{2} \\
& \left\{3^{\beta} \cdot 5 \cdot 7^{2} \cdot 11\right\}_{\beta=0,1,2}\left\{2 \cdot 3^{\beta} \cdot 5 \cdot 7^{2} \cdot 11\right\}_{\beta=0,1,2}^{2} \\
& \text { similar to treatment of }\{2 \cdot 3 \cdot 5 \cdot 7 \cdot 11\}^{10} \\
& \left\{3^{\beta} \cdot 5^{k} \cdot 7 \cdot 11\right\}_{\beta=0,1,2}\left\{2 \cdot 3^{\beta} \cdot 5^{k} \cdot 7 \cdot 11\right\}_{\beta=0,1,2}^{2} \\
& \left\{3^{\beta} \cdot 5^{k} \cdot 7^{2} \cdot 11\right\}_{\beta=0,1,2}\left\{2 \cdot 3^{\beta} \cdot 5^{k} \cdot 7^{2} \cdot 11\right\}_{\beta=0,1,2}^{2}\left\{2 \cdot 3 \cdot 5^{k} \cdot 7 \cdot 11 \cdot 37\right\}^{74} \\
& \left\{2 \cdot 3 \cdot 5^{\gamma} \cdot 7 \cdot 11 \cdot 37\right\}_{\gamma=1,2}, \cdots, 74 \\
& \text { treated in a similar way to }\{2 \cdot 3 \cdot 7 \cdot 11\}^{11} \\
& \left\{3^{\beta} \cdot 7 \cdot 11^{k}\right\}_{\beta=0,1}\left\{2 \cdot 3^{\beta} \cdot 7 \cdot 11^{k}\right\}_{\beta=0,1}^{2}\left\{2 \cdot 3^{2} \cdot 7 \cdot 11^{k}\right\}^{3} \\
& \left\{2 \cdot 3 \cdot 5 \cdot 7 \cdot 11^{k}\right\}^{10}\left\{2 \cdot 3 \cdot 7^{2} \cdot 11^{k}\right\}^{7}\left\{2 \cdot 3 \cdot 7 \cdot 11^{k} \cdot 37\right\}^{37} \\
& \left\{2 \cdot 3 \cdot 7 \cdot 11^{8} \cdot 37\right\}_{\delta-1,2, \cdots, 37}
\end{aligned}
$$

Step 3. Here $\gamma=1$ or 2.

$$
\begin{aligned}
& \left\{2^{2} \cdot 3 \cdot 7^{\gamma} \cdot 13\right\}^{13} \\
& \left\{2^{\alpha} \cdot 3^{\beta} \cdot 7^{\gamma} \cdot 13\right\}_{\alpha=0,1 ; \beta=0,1}\left\{2^{2} \cdot 3^{\beta} \cdot 7^{\gamma} \cdot 13\right\}_{\beta=0,1}^{2}\left\{2^{2} \cdot 3^{2} \cdot 7^{\gamma} \cdot 13\right\}^{6} \\
& \left\{2^{2} \cdot 3 \cdot 5 \cdot 7^{\gamma} \cdot 13\right\}^{10}\left\{2^{2} \cdot 3 \cdot 7^{\gamma} \cdot 11 \cdot 13\right\}^{11} \\
& \left\{2^{2} \cdot 3^{2} \cdot 7^{\gamma} \cdot 13\right\}^{6} \\
& \left\{2^{\alpha} \cdot 3^{2} \cdot 7^{\gamma} \cdot 13\right\}_{\alpha=0,1}\left\{2^{2} \cdot 3^{2} \cdot 7^{\gamma} \cdot 13\right\}^{2}\left\{2^{2} \cdot 3^{3} \cdot 7^{\gamma} \cdot 13\right\}^{8} \\
& \left\{2^{2} \cdot 3^{k} \cdot 7^{\gamma} \cdot 13\right\}^{6} \\
& \left\{2^{2} \cdot 3^{k} \cdot 7^{\gamma} \cdot 13 \cdot 37\right\}^{74} \\
& \left\{2^{2} \cdot 3 \cdot 5 \cdot 7^{\gamma} \cdot 13\right\}^{10} \\
& \left\{2^{2} \cdot 3^{2} \cdot 5 \cdot 7^{\gamma} \cdot 13\right\}^{6} \\
& \text {............ } \\
& \left\{2^{2} \cdot 3^{k} \cdot 5 \cdot 7^{\gamma} \cdot 13\right\}^{6} \\
& \left\{2^{2} \cdot 3^{k} \cdot 5 \cdot 7^{\gamma} \cdot 13 \cdot 37\right\}^{74} \\
& \left\{2^{\alpha} \cdot 3^{k} \cdot 7^{\gamma} \cdot 13\right\}_{\alpha=0,1}\left\{2^{2} \cdot 3^{k} \cdot 7^{\gamma} \cdot 13\right\}^{2}\left\{2^{2} \cdot 3^{k} \cdot 7^{\gamma} \cdot 13 \cdot 37\right\}^{74} \\
& \left\{2^{2} \cdot 3^{\beta} \cdot 7^{\gamma} \cdot 13 \cdot 37\right\}_{\beta=1,2}, \cdots, 74 \\
& \left\{2^{\alpha} \cdot 3^{\beta} \cdot 5 \cdot 7^{\gamma} \cdot 13\right\}_{\alpha=0,1 ; \beta=0,1}\left\{2^{2} \cdot 3^{\beta} \cdot 5 \cdot 7^{\gamma} \cdot 13\right\}_{\beta-0,1}^{2} \\
& \left\{2^{2} \cdot 3^{2} \cdot 5 \cdot 7^{\gamma} \cdot 13\right\}^{6} \\
& \left\{2^{\alpha} \cdot 3^{2} \cdot 5 \cdot 7^{\gamma} \cdot 13\right\}_{\alpha=0,1}\left\{2^{2} \cdot 3^{2} \cdot 5 \cdot 7^{\gamma} \cdot 13\right\}^{2}\left\{2^{2} \cdot 3^{3} \cdot 5 \cdot 7^{\gamma} \cdot 13\right\}^{\sigma} \\
& \left\{2^{\alpha} \cdot 3^{k} \cdot 5 \cdot 7^{\gamma} \cdot 13\right\}_{\alpha=0,1}\left\{2^{2} \cdot 3^{k} \cdot 5 \cdot 7^{\gamma} \cdot 13\right\}^{2} \\
& \left\{2^{2} \cdot 3^{k} \cdot 5 \cdot 7^{\gamma} \cdot 13 \cdot 37\right\}^{74} \\
& \left\{2^{2} \cdot 3 \cdot 7^{\gamma} \cdot 11 \cdot 13\right\}^{11} \\
& \left\{2^{2} \cdot 3^{\beta} \cdot 5 \cdot 7^{\gamma} \cdot 13 \cdot 37\right\}_{\beta-1,2, \cdots, 74} \\
& \left\{2^{\alpha} \cdot 3^{\beta} \cdot 7^{\gamma} \cdot 11 \cdot 13\right\}_{\alpha=0,1 ; \beta=0,1}\left\{2^{2} \cdot 3^{\beta} \cdot 7^{\gamma} \cdot 11 \cdot 13\right\}_{\beta=0,1}^{2} \\
& \left\{2^{2} \cdot 3^{2} \cdot 7^{\gamma} \cdot 11 \cdot 13\right\}^{6}\left\{2^{2} \cdot 3 \cdot 5 \cdot 7^{\gamma} \cdot 11 \cdot 13\right\}^{5} \\
& \left\{2^{2} \cdot 3 \cdot 5 \cdot 7^{\gamma} \cdot 11 \cdot 13\right\}^{5} \\
& \left\{2^{\alpha} \cdot 3^{\beta} \cdot 5 \cdot 7^{\gamma} \cdot 11 \cdot 13\right\}_{\alpha=0,1,2 ; \beta-0,1} \\
& \left\{2^{2} \cdot 3^{2} \cdot 7^{\gamma} \cdot 11 \cdot 13\right\}^{6} \\
& \text { treated in a similar way to }\left\{2^{2} \cdot 3^{2} \cdot 5 \cdot 7^{\gamma} \cdot 13\right\}^{\circ}
\end{aligned}
$$

Proof of Theorem 9. 


\begin{tabular}{l|l}
$\left\{2^{2} \cdot 3\right\}=\left\{2^{2} \cdot 3 \cdot 11\right\}^{11}$ & $\begin{array}{l}\{2 \cdot 11\}\{3 \cdot 11\}\{2 \cdot 3 \cdot 11\}\left\{2^{2} \cdot 3^{\beta} \cdot 11\right\}_{\beta-0,1}^{2} \\
\left\{2^{2} \cdot 3^{2} \cdot 11\right\}^{6}\left\{2^{2} \cdot 3 \cdot 5 \cdot 11\right\}^{10}\end{array}$ \\
$\left\{2^{2} \cdot 3^{2} \cdot 11\right\}^{6}$ & $\left\{2^{\alpha} \cdot 3^{2} \cdot 11\right\}_{\alpha=0,1}\left\{2^{2} \cdot 3^{2} \cdot 11\right\}^{2}\left\{2^{2} \cdot 3^{3} \cdot 11\right\}^{6}$ \\
$\cdots \cdots \cdots \cdots$ & $\left\{2^{2} \cdot 3^{k} \cdot 11\right\}^{6}$ \\
$\left\{2^{2} \cdot 3^{k} \cdot 11 \cdot 37\right\}^{74}$ & $\left\{2^{\alpha} \cdot 3^{k} \cdot 11\right\}_{\alpha=0,1}\left\{2^{2} \cdot 3^{k} \cdot 11\right\}^{2}\left\{2^{2} \cdot 3^{k} \cdot 11 \cdot 37\right\}^{74}$ \\
$\left\{2^{2} \cdot 3 \cdot 5 \cdot 11\right\}^{10}$ & $\left\{2^{2} \cdot 3^{\beta} \cdot 11 \cdot 37\right\}_{\beta-1,2} \cdots, 74$ \\
$\left\{2^{2} \cdot 3^{2} \cdot 5 \cdot 11\right\}^{6}$ & $\left\{2^{\alpha} \cdot 3^{\beta} \cdot 5 \cdot 11\right\}_{\alpha=0,1 ; \beta-0,1}\left\{2^{2} \cdot 3^{\beta} \cdot 5 \cdot 11\right\}_{\beta-0,1}^{2}\left\{2^{2} \cdot 3^{2} \cdot 5 \cdot 11\right\}^{6}$ \\
$\cdots \cdots \cdots$ & $\left\{2^{\alpha} \cdot 3^{2} \cdot 5 \cdot 11\right\}_{\alpha=0,1}\left\{2^{2} \cdot 3^{2} \cdot 5 \cdot 11\right\}^{2}\left\{2^{2} \cdot 3^{3} \cdot 5 \cdot 11\right\}^{6}$ \\
$\left\{2^{2} \cdot 3^{k} \cdot 5 \cdot 11\right\}^{6}$ & $\left\{2^{\alpha} \cdot 3^{k} \cdot 5 \cdot 11\right\}_{\alpha=0,1}\left\{2^{\alpha} \cdot 3^{k} \cdot 5 \cdot 11\right\}^{2}\left\{2^{2} \cdot 3^{k} \cdot 5 \cdot 11 \cdot 37\right\}^{74}$ \\
$\left\{2^{2} \cdot 3^{k} \cdot 5 \cdot 11 \cdot 37\right\}^{74}$ & $\left\{2^{2} \cdot 3^{\beta} \cdot 5 \cdot 11 \cdot 37\right\}_{\beta-1,2, \cdots, 74}$
\end{tabular}

Proof of Theorem 10.

\begin{tabular}{|c|c|}
\hline$\left\{2 \cdot 3^{2}\right\}=\left\{2 \cdot 3^{2} \cdot 13\right\}^{13}$ & $\left\{3^{\alpha} \cdot 13\right\}_{\alpha-1,2}\left\{2 \cdot 3^{\alpha} \cdot 13\right\}_{\alpha=0,1,2}^{2}\left\{2 \cdot 3^{3} \cdot 13\right\}^{3}$ \\
\hline & $\left\{2 \cdot 3^{2} \cdot 5 \cdot 13\right\}^{10}\left\{2 \cdot 3^{2} \cdot 11 \cdot 13\right\}^{11}\left\{2 \cdot 3^{2} \cdot 13^{2}\right\}^{13}$ \\
\hline$\left\{2 \cdot 3^{3} \cdot 13\right\}^{3}$ & $\left\{3^{3} \cdot 13\right\}\left\{2 \cdot 3^{3} \cdot 13\right\}^{2}$ \\
\hline$\left\{2 \cdot 3^{2} \cdot 5 \cdot 13\right\}^{10}$ & $\left\{3^{\alpha} \cdot 5 \cdot 13\right\}_{\alpha=0.1,2}\left\{2 \cdot 3^{\alpha} \cdot 5 \cdot 13\right\}_{\alpha=0,1,2}^{2}\left\{2 \cdot 3^{3} \cdot 5 \cdot 13\right\}^{3}$ \\
\hline$\left\{2 \cdot 3^{3} \cdot 5 \cdot 13\right\}^{3}$ & $\left\{3^{3} \cdot 5 \cdot 13\right\}\left\{2 \cdot 3^{3} \cdot 5 \cdot 13\right\}^{2}$ \\
\hline$\left\{2 \cdot 3^{2} \cdot 11 \cdot 13\right\}^{11}$ & $\left\{3^{\alpha} \cdot 11 \cdot 13\right\}_{\alpha=0,1,2}\left\{2 \cdot 3^{\alpha} \cdot 11 \cdot 13\right\}_{\alpha=0,1,2}^{2}\left\{2 \cdot 3^{3} \cdot 11 \cdot 13\right\}^{3}$ \\
\hline & $\left\{2 \cdot 3^{2} \cdot 5 \cdot 11 \cdot 13\right\}^{5}$ \\
\hline$\left\{2 \cdot 3^{3} \cdot 11 \cdot 13\right\}^{3}$ & $\left\{3^{3} \cdot 11 \cdot 13\right\}\left\{2 \cdot 3^{3} \cdot 11 \cdot 13\right\}^{2}$ \\
\hline$\left\{2 \cdot 3^{2} \cdot 5 \cdot 11 \cdot 13\right\}^{5}$ & $\left\{3^{\alpha} \cdot 5 \cdot 11 \cdot 13\right\}_{\alpha=0,1,2}\left\{2 \cdot 3^{2} \cdot 5 \cdot 11 \cdot 13\right\}^{2}$ \\
\hline$\left\{2 \cdot 3^{2} \cdot 13^{2}\right\}^{13}$ & $\left\{3^{\alpha} \cdot 13^{2}\right\}_{\alpha=0,1,2}\left\{2 \cdot 3^{\alpha} \cdot 13^{2}\right\}_{\alpha=0,1,2}^{2}\left\{2 \cdot 3^{3} \cdot 13^{2}\right\}^{3}$ \\
\hline & $\left\{2 \cdot 3^{2} \cdot 5 \cdot 13^{2}\right\}^{10}\left\{2 \cdot 3^{2} \cdot 11 \cdot 13^{2}\right\}^{11}$ \\
\hline$\left\{2 \cdot 3^{3} \cdot 13^{2}\right\}^{3}$ & $\left\{3^{3} \cdot 13^{2}\right\}\left\{2 \cdot 3^{3} \cdot 13^{2}\right\}^{2}$ \\
\hline$\left\{2 \cdot 3^{2} \cdot 5 \cdot 13^{2}\right\}^{10}$ & treatment similar to that of $\left\{2 \cdot 3^{2} \cdot 5 \cdot 13\right\}^{10}$ \\
\hline$\left\{2 \cdot 3^{2} \cdot 11 \cdot 13^{2}\right\}^{11}$ & treatment similar to that of $\left\{2 \cdot 3^{2} \cdot 11 \cdot 13\right\}^{11}$ \\
\hline
\end{tabular}

Proof of Theorem 11.

$$
\begin{array}{l|l}
\left\{2^{3} \cdot 3\right\}=\left\{2^{3} \cdot 3 \cdot 17\right\}^{17} & \left\{2^{\alpha} \cdot 17\right\}_{\alpha-1,2}\left\{2^{\alpha} \cdot 3 \cdot 17\right\}_{\alpha=0,1,2}\left\{2^{3} \cdot 3^{\beta} \cdot 17\right\}_{\beta-0,1}^{2} \\
& \left\{2^{3} \cdot 3^{2} \cdot 17\right\}^{6}\left\{2^{3} \cdot 3 \cdot 5 \cdot 17\right\}^{15}\left\{2^{3} \cdot 3 \cdot 7 \cdot 17\right\}^{7} \\
& \left\{2^{3} \cdot 3 \cdot 11 \cdot 17\right\}^{11}\left\{2^{3} \cdot 3 \cdot 13 \cdot 17\right\}^{13} \\
& \left\{2^{\alpha} \cdot 3^{2} \cdot 17\right\}_{\alpha=0,1,2}\left\{2^{3} \cdot 3^{2} \cdot 17\right\}^{2}\left\{2^{3} \cdot 3^{3} \cdot 17\right\}^{3} \\
\left\{2^{3} \cdot 3^{2} \cdot 17\right\}^{6} & \left\{2^{2} \cdot 3^{3} \cdot 17\right\}\left\{2^{3} \cdot 3^{3} \cdot 17\right\}^{2} \\
\left\{2^{3} \cdot 3^{3} \cdot 17\right\}^{3} &
\end{array}
$$




$$
\begin{array}{l|l}
\left\{2^{3} \cdot 3 \cdot 5 \cdot 17\right\}^{15} & \begin{array}{l}
\left\{2^{\alpha} \cdot 3^{\beta} \cdot 5 \cdot 17\right\}_{\alpha=0,1,2 ; \beta=0,1}\left\{2^{3} \cdot 3^{\beta} \cdot 5 \cdot 17\right\}_{\beta=0,1}^{2} \\
\left\{2^{3} \cdot 3^{2} \cdot 5 \cdot 17\right\}^{\beta}\left\{2^{3} \cdot 3 \cdot 5^{2} \cdot 17\right\}^{15}
\end{array} \\
\begin{array}{l}
\left\{2^{3} \cdot 3^{2} \cdot 5 \cdot 17\right\}^{6} \\
\left\{2^{3} \cdot 3 \cdot 5^{2} \cdot 17\right\}^{15}
\end{array} & \begin{array}{l}
\left\{2^{\alpha} \cdot 3^{\beta} \cdot 5 \cdot 17\right\}_{\alpha=0,1,2 ; \beta=2,3}\left\{2^{3} \cdot 3^{2} \cdot 5 \cdot 17\right\}^{2} \\
\text { treatment similar to }\left\{2^{3} \cdot 3 \cdot 5 \cdot 17\right\}^{15}
\end{array} \\
\begin{array}{l}
\left\{2^{3} \cdot 3 \cdot 5^{k} \cdot 17 \cdot 37\right\}^{111} \\
\left\{2^{3} \cdot 3 \cdot 7 \cdot 17\right\}^{7}
\end{array} & \begin{array}{l}
\left\{2^{3} \cdot 3 \cdot 5^{\gamma} \cdot 17 \cdot 37\right\}_{\gamma=1,2, \cdots, 111} \\
\left\{2^{\alpha} \cdot 3^{\beta} \cdot 7 \cdot 11\right\}_{\alpha=0,1,2,3 ; \beta=0,1} \\
\left\{2^{3} \cdot 3 \cdot 11 \cdot 17\right\}^{11}
\end{array} \\
\left\{2^{3} \cdot 3 \cdot 13 \cdot 17\right\}^{13} & \left\{2^{\alpha} \cdot 3^{\beta} \cdot 11 \cdot 17\right\}_{\alpha=0,1,2 ; \beta=0,1,2}\left\{2^{3} \cdot 3^{\beta} \cdot 11 \cdot 17\right\}_{\beta=0,1}^{2} \\
& \left\{2^{\alpha} \cdot 3^{\beta} \cdot 13 \cdot 17\right\}_{\alpha=0,1,2 ; \beta=0,1,2}\left\{2^{3} \cdot 3^{\beta} \cdot 13 \cdot 17\right\}_{\beta=0,1}^{2} \\
& \left\{2^{3} \cdot 3 \cdot 5 \cdot 13 \cdot 17\right\}^{10} \\
\left\{2^{3} \cdot 3 \cdot 5 \cdot 13 \cdot 17\right\}^{10} & \left\{2^{\alpha} \cdot 3^{\beta} \cdot 5 \cdot 13 \cdot 17\right\}_{\alpha=0,1,2 ; \beta=0,1}\left\{2^{3} \cdot 3^{\beta} \cdot 5 \cdot 13 \cdot 17\right\}_{\beta=0,1}^{2}
\end{array}
$$

Proof of Theorem 12.

$$
\begin{array}{l|l}
\left\{2^{3} \cdot 3\right\}=\left\{2^{3} \cdot 3 \cdot 19\right\}^{19} & \begin{array}{l}
\{3 \cdot 19\}\left\{2^{\alpha} \cdot 3^{\beta} \cdot 19\right\}_{\alpha=1,2 ; \beta=0,1}\left\{2^{3} \cdot 3^{\beta} \cdot 19\right\}_{\beta=0,1}^{2} \\
\left\{2^{3} \cdot 3^{2} \cdot 19\right\}^{6}\left\{2^{3} \cdot 3 \cdot 5 \cdot 19\right\}^{15}\left\{2^{3} \cdot 3 \cdot 7 \cdot 19\right\}^{14}
\end{array} \\
\left\{2^{3} \cdot 3^{2} \cdot 19\right\}^{6} & \begin{array}{l}
\left\{2^{3} \cdot 3 \cdot 11 \cdot 19\right\}^{11}\left\{2^{3} \cdot 3 \cdot 13 \cdot 19\right\}^{13}\left\{2^{3} \cdot 3 \cdot 17 \cdot 19\right\}^{17} \\
\left\{2^{\alpha} \cdot 3^{\beta} \cdot 19\right\}_{\alpha=0,1,2 ; \beta=2,3}\left\{2^{3} \cdot 3^{2} \cdot 19\right\}^{2}
\end{array} \\
\left\{2^{3} \cdot 3 \cdot 5: 19\right\}^{15} & \begin{array}{l}
\text { treatment similar to that of }\left\{2^{3} \cdot 3 \cdot 5 \cdot 17\right\}^{15} \\
\text { in proof of Theorem } 11
\end{array} \\
\left\{2^{3} \cdot 3 \cdot 7 \cdot 19\right\}^{14} & \begin{array}{l}
\left\{2^{\alpha} \cdot 3^{\beta} \cdot 7 \cdot 19\right\}_{\alpha=0,1,2 ; \beta=0,1}\left\{2^{3} \cdot 3^{\beta} \cdot 7 \cdot 19\right\}_{\beta=0,1}^{2} \\
\left\{2^{3} \cdot 3^{2} \cdot 7 \cdot 19\right\}^{6}\left\{2^{3} \cdot 3 \cdot 5 \cdot 7 \cdot 19\right\}^{10}
\end{array} \\
\left\{2^{3} \cdot 3^{2} \cdot 7 \cdot 19\right\}^{6} & \left\{2^{\alpha} \cdot 3^{\beta} \cdot 7 \cdot 19\right\}_{\alpha=0,1,2 ; \beta=2,3}\left\{2^{3} \cdot 3^{2} \cdot 5 \cdot 7 \cdot 19\right\}^{2} \\
\left\{2^{3} \cdot 3 \cdot 5 \cdot 7 \cdot 19\right\}^{10} & \left\{2^{\alpha} \cdot 3^{\beta} \cdot 5 \cdot 7 \cdot 19\right\}_{\alpha=0,1,2 ; \beta=0,1}\left\{2^{3} \cdot 3^{\beta} \cdot 5 \cdot 7 \cdot 19\right\}_{\beta=0,1}^{2} \\
\left\{2^{3} \cdot 3 \cdot 11 \cdot 19\right\}^{11} & \left\{2^{\alpha} \cdot 3^{\beta} \cdot 11 \cdot 19\right\}_{\alpha=0,1,2 ; \beta=0,1,2}\left\{2^{3} \cdot 3^{\beta} \cdot 11 \cdot 19\right\}_{\beta=0,1}^{2} \\
\left\{2^{3} \cdot 3 \cdot 13 \cdot 19\right\}^{13} & \left\{2^{\alpha} \cdot 3^{\beta} \cdot 13 \cdot 19\right\}_{\alpha=0,1,2 ; \beta=0,1,2}\left\{2^{3} \cdot 3^{\beta} \cdot 13 \cdot 19\right\}_{\beta=0,1}^{2} \\
& \left\{2^{\alpha} \cdot 3^{\beta} \cdot 5 \cdot 13 \cdot 19\right\}_{\alpha=0,1,2 ; \beta=0,1}\left\{2^{3} \cdot 3^{\beta} \cdot 5 \cdot 13 \cdot 19\right\}_{\beta=0,1}^{2} \\
& 19 Q_{7}=\left\{19 x ; x \in Q_{7}\right\}
\end{array}
$$

\section{Proof of Theorem 13.}

$\left\{2^{3} \cdot 3\right\}=\left\{2^{3} \cdot 3 \cdot 23\right\}^{23}$
$\left\{2^{3} \cdot 3^{2} \cdot 23\right\}^{6}$
$\left\{2^{3} \cdot 3 \cdot 5 \cdot 23\right\}^{15}$

$\left\{2^{\alpha} \cdot 3^{\beta} \cdot 23\right\}_{\alpha=0,1,2 ; \beta=0,1}\left\{2^{3} \cdot 3^{\beta} \cdot 23\right\}_{\beta=0,1}^{2}\left\{2^{3} \cdot 3^{2} \cdot 23\right\}^{6}$

$\left\{2^{3} \cdot 3 \cdot 5 \cdot 23\right\}^{15}\left\{2^{3} \cdot 3 \cdot p \cdot 23\right\}_{p=11,13,17,19}^{p}$ $\left\{2^{3} \cdot 3 \cdot 7 \cdot 23\right\}^{14}\left\{2^{3} \cdot 3 \cdot 23 \cdot 29\right\}^{29}\left\{2^{3} \cdot 3 \cdot 23 \cdot 31\right\}^{31}$

similar to treatment of $\left\{2^{3} \cdot 3^{2} \cdot 19\right\}^{6}$ similar to treatment of $\left\{2^{3} \cdot 3 \cdot 5 \cdot 19\right\}^{15}$ 


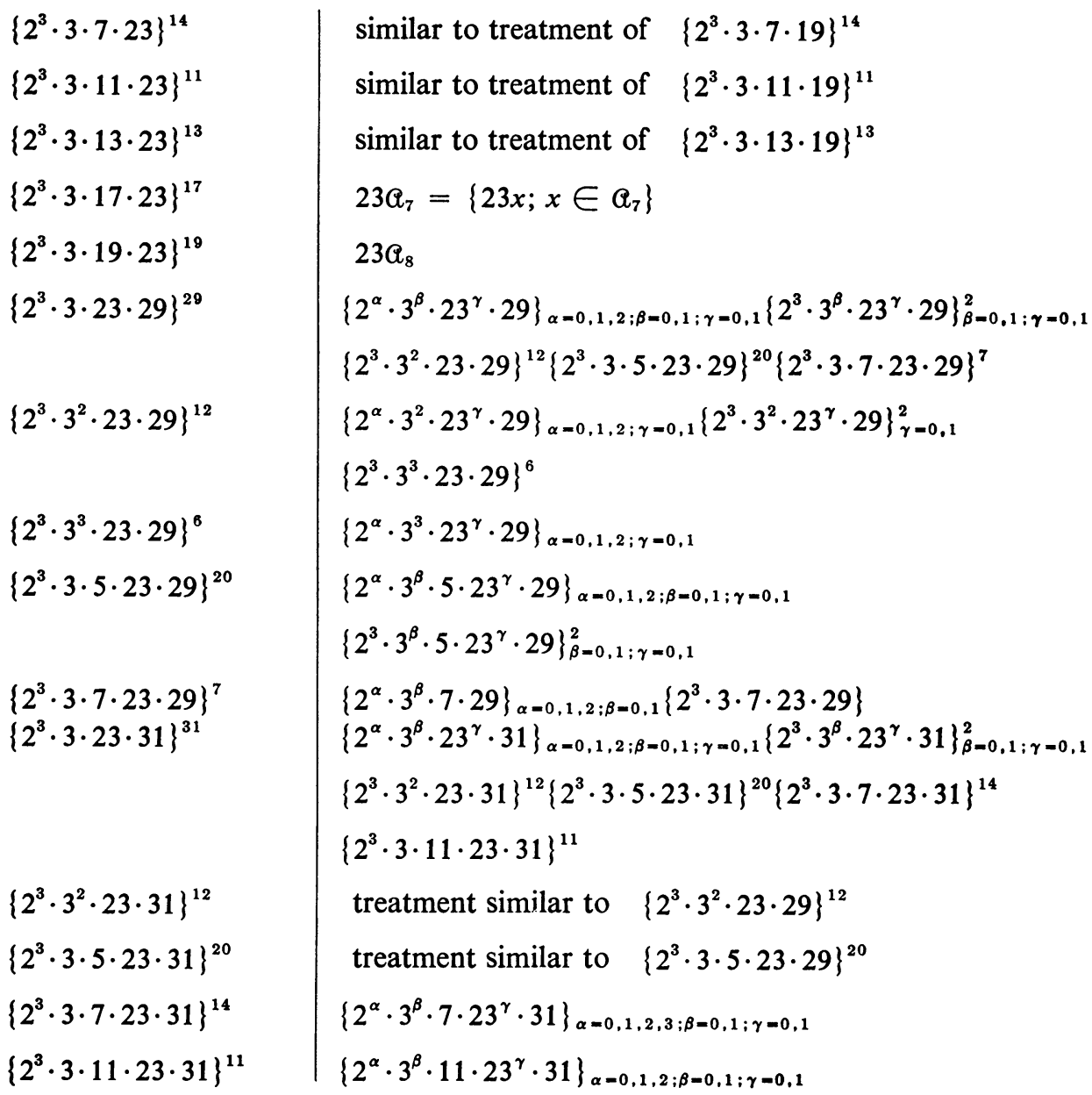

Department of Mathematics University of British Columbia Vancouver 8, B. C., Canada

1. P. Erdös, Quelques Problèmes de la Théorie des Nombres, Monographies de L'Enseignement Mathématique, no. 6, L'Enseignement Mathématique, Université de Genève, 1963, pp. 81-135. MR 28 \#2070.

2. R. F. ChURChHOUSE, "Covering sets and systems of congruences," in Computers in Mathematical Research, North-Holland, Amsterdam, 1968, pp. 20-36. MR 39 \#1399.

3. C. E. KRUkenberg, Ph.D. Thesis, University of Illinois, Urbana-Champaign, Ill., 1971 , pp. $38-77$. 